\title{
Análisis Exploratorio de las Percepciones sobre los Estilos de Liderazgo, el Clima Académico y la Calidad de la Formación de Pregrado
}

\author{
Andrés Bernasconi ${ }^{(1)}$ y Emilio Rodríguez-Ponce ${ }^{(2)^{*}}$ \\ (1) Pontificia Universidad Católica de Chile, Fac. de Educación, A. Vicuña Mackena 4860, Macul, \\ Santiago - Chile. \\ (2) Universidad de Tarapacá, Instituto de Alta Investigación. Casilla 26. Arica - Chile. \\ * Autor a quien debe ser dirigida la correspondencia
}

Recibido Ago. 23, 2017; Aceptado Oct. 23, 2017; Versión final Dic. 21, 2017, Publicado Jun. 2018

\begin{abstract}
Resumen
En esta investigación se explora las percepciones de directivos universitarios de unidades académicas, respecto de la potencial relación entre: estilos de liderazgo, clima organizativo y calidad de carreras de pregrado en una muestra de 40 unidades académicas pertenecientes a cuatro universidades en Chile. Los resultados muestran que existe evidencia para relacionar la calidad de las carreras negativamente con el estilo de liderazgo laissez faire (Test $t=-2,418 ; p<0,021$ ) y positivamente con el clima orientado a la excelencia (Test $t=6,647 ; p<0,01$ ). Ambas variables explican, en conjunto, el $67,3 \%$ de la varianza en la calidad de las carreras de pregrado. A su vez, el clima orientado a la excelencia depende positivamente del estilo de liderazgo transformacional (Test $t=10,729 ; p<0,01$ ), por lo que el impacto de este estilo sobre la calidad de las carreras es indirecto, pero altamente significativo en la muestra analizada. Se discuten los hallazgos e implicancias resaltando la importancia de las relaciones encontradas.
\end{abstract}

\section{Exploratory Analysis about the Perceived Leadership Styles, Academic Climate and Quality of Undergraduates Programs}

\begin{abstract}
This research aims to explore the relationship between: leadership styles, organizational climate in academic units and the quality of undergraduate programs. For this purpose, a sample of 40 academic units belonging to 4 different universities in Chile were considered. The results of the research show that there is evidence to correlate the quality of the degrees negatively with the laissez-faire style $($ Test $t=-2.418 ; p<0.021)$ and positively with the excellence-oriented climate $(T e s t t=6.647 ; p<0.01)$. Both variables jointly explain $67.3 \%$ of the variance in the quality of undergraduate programs. In turn, the excellence-oriented climate positively depends on the transformational leadership style (Test $t=10,729, p<0.01$ ), so its impact on the quality of university degrees is indirect but highly significant in the analysed sample. Finally, the scope and implications of the findings are discussed, highlighting the importance of the results.
\end{abstract}

Keywords: educational quality, academic degrees, organizational climate, leadership styles 


\section{INTRODUCCIÓN}

En la sociedad actual, la fuente fundamental de la ventaja competitiva de las naciones, de las organizaciones y de las personas reside en el conocimiento. Ciertamente, la dicotomía entre capital y trabajo es conceptualmente superada y no constituye el problema central de la generación de la riqueza, ya que tanto el capital como el trabajo son requeridos para la producción de bienes, pero el conocimiento es la base o esencia de la nueva economía. Así, la innovación y la productividad son expresiones concretas del conocimiento individual y del conocimiento colectivo que, a su vez, impactan sobre el desarrollo económico y social (Castells, 2011). Una parte primordial de ese conocimiento deriva del capital humano avanzado que dispone la sociedad y que se genera en las instituciones de educación superior. A su turno, el nivel de preparación intelectual de las personas se asocia indisolublemente con sus futuras posibilidades de inclusión en la sociedad y en el mundo del trabajo (Barro y Lee, 2013). De este modo, la calidad de la formación universitaria constituye uno de los aspectos de mayor importancia para influir sobre la capacidad competitiva de los países y sus organizaciones públicas y privadas. Por lo demás, la calidad con que se imparten las carreras universitarias resulta ser la expresión más concreta, para los estudiantes, de la formación recibida y un determinante para su potencial inserción y posterior desarrollo como profesional (Hanushek, 2013).

Ahora bien, la calidad de los procesos de formación en los estudios universitarios tiene una serie de variables explicativas que han sido analizadas en el estado del arte. En este sentido se ha descubierto que la calidad del cuerpo académico es un factor clave que impacta en el proceso de enseñanza, aprendizaje y en los resultados académicos (Duque, 2014). Asimismo, la infraestructura, laboratorios, equipamiento y los recursos educativos inciden en los procesos de aprendizaje y en sus resultados (Horn y Lee, 2016). Naturalmente, la calidad que alcancen los graduados universitarios depende también de su potencial académico e intelectual medido por pruebas de selección al ingreso al sistema de educación superior (Dickinson y Adelson, 2016). En este mismo contexto, la evidencia internacional reconoce que los estilos de liderazgo pueden incidir en: el clima, la cultura y el desempeño académico; o incluso en el nivel de logro de los estudiantes y en el compromiso de los profesores; impactando de este modo en los procesos de enseñanza y en los resultados académicos (Ersozlu y Saklan, 2016).

Sin embargo, las investigaciones que vinculan los estilos de liderazgo con calidad de las titulaciones son escasas y prácticamente inexistentes en los países en vías de desarrollo. Por lo mismo, explorar dicho vínculo parece no sólo plausible sino que necesario si es que se pretende avanzar en mejorar la calidad de la formación universitaria, cuestión que, además, emerge como un desafío esencial para una inserción exitosa de los países, las organizaciones y las personas, en la sociedad del conocimiento. Más aún, en esta investigación se pretende explorar la relación entre estilos de liderazgo, clima organizacional y calidad de las carreras universitarias, ya que se ha encontrado evidencia de la relación entre estilos de liderazgo y clima organizativo (Alonderiene y Majauskaite, 2016), ya que por medio de la identificación del clima organizacional que domina una organización, los líderes podrían ser capaces de optimizar el desempeño mediante la identificación de las fortalezas y debilidades (Belias et al., 2017); sin embargo, los estudios que relacionan el clima organizativo con la calidad de las titulaciones universitarias son muy escasos.

En este estudio específico se procura identificar los impactos directos e indirectos de los estilos de liderazgo sobre la calidad de las carreras universitarias, superándose así la problemática asociada a ignorar variables intermedias que puedan ser relevantes, como por ejemplo lo puede ser el clima organizativo en las unidades académicas, y cuya incorporación podría proveer una mejor comprensión de la realidad. El objetivo referido a explorar las relaciones señaladas precedentemente permite trabajar en pequeña escala para identificar mediante datos empíricos aspectos fundamentales que proporcionen una visión general, de tipo aproximativo, de un determinado fenómeno o realidad. Por lo tanto, el análisis exploratorio es pertinente y necesario en esta investigación toda vez que la temática no ha sido suficientemente estudiada en los países emergentes, las relaciones exploradas no han sido indagadas en profundidad e incluso las medidas podrían requerir una validación y demostrar su fiabilidad para estas relaciones específicas. En consecuencia, en esta investigación se procura específicamente validar las variables y sus medidas, analizar su fiabilidad, y establecer una primera mirada acerca de la pertinencia de las relaciones entre las variables estudiadas (Long et al., 2012).

Desde luego, la idea de que la educación sirve sólo o principalmente al propósito de aumentar el capital humano, para una mayor productividad, y de ese modo, mayores ingresos, también ha sido discutida desde perspectivas de filosofía moral (con mayores consecuencias, por Nussbaum y Sen, 1993 y, en versiones más recientes de estas argumentaciones, por ejemplo, en la colección de trabajos reunidos en Vally y Motala, 2014). Boni y Walker (2016), por su parte, han volcado esta cuestión al campo más acotado de la educación universitaria. Estas perspectivas desafían la conceptualización de la educación como medio para el progreso material, y enfatizan más bien su rol liberador y democratizante, de desarrollo personal, y de fomento de virtudes ciudadanas. 
Compartimos la idea de que la educación superior no es solo formación para el trabajo, así como la proposición, empíricamente bien establecida, de que las personas eligen educarse por razones que exceden el puro cálculo de costo-beneficio.

\section{MARCO DE REFERENCIA}

El marco de referencia se presenta en tres secciones, referidas a los estilos de liderazgo, al clima de las unidades académicas y a la calidad de las carreras universitarias.

\section{Estilos de liderazgo}

El líder tiene un papel fundamental en un equipo u organización ya que es quien influye para que los miembros de dicho equipo o de la organización lleven a cabo las tareas requeridas para conseguir los objetivos propuestos. Así las cosas, el líder debe lograr mediante un proceso de influencia que otros realicen, con él o por él, lo que se desea y se espera de ellos. Dicho de modo claro, el liderazgo se refiere a la capacidad de influir, incidir o inducir a los grupos o individuos al interior de un equipo o una organización para lograr una determinada actuación o comportamiento en favor de alcanzar los objetivos o metas institucionales (Solajà et al., 2016). La evidencia internacional ha mostrado la relevancia de los estilos de liderazgo para explicar los resultados de los equipos de trabajo (Mukherjee, 2016), así como también para influir en el desempeño del sector público de de las organizaciones (Zeb et al., 2015). Ahora bien, en educación superior hay hallazgos relevantes que ponen de manifiesto la importancia del liderazgo para generar compromiso en los académicos (Henkel, 2016), alcanzar mayor satisfacción en el trabajo (Alonderiene y Majauskaite, 2016), lograr mejores resultados individuales (Jyoti y Bhau, 2016), e institucionales (Wahab et al., 2016). Existen múltiples formas de tipificar los estilos de liderazgo. No obstante, en los últimos años se ha consolidado la teoría de Bass y Avolio que identifica tres dimensiones para estudiar el liderazgo: (1) liderazgo transformacional; (2) liderazgo transaccional; (3) laissez faire (Pedraja-Rejas et al., 2016). Los principales rasgos distintivos de estas dimensiones se sintetizan seguidamente:

El Liderazgo Transformacional se singulariza por cuanto el líder genera propósitos y metas que son desafiantes, las que se procuran alcanzar como institución o como equipo, para lo cual se empodera a los seguidores y se construye un clima moral basado en los valores institucionales y en destacar la relevancia del aporte de cada miembro del equipo a los propósitos institucionales (por ejemplo, Cheng et al., 2016). Las características que se asocian a los líderes transformacionales son: lograr una influencia idealizada basada en el carisma del líder, el que genera confianza en los seguidores quienes lo admiran, respetan e imitan (Pedraja y Rodríguez 2014); generar una motivación inspiracional construyendo la perspectiva de un futuro deseable, alcanzable y proporcionando significado y valor intrínseco al trabajo de las personas en un clima de colaboración y respeto (Pedraja-Rejas et al., 2016); lograr la estimulación intelectual fomentando la creatividad, la innovación y desafiando las formas tradicionales de hacer la tarea (Araneda-Guirriman et al. 2016); sin perder de vista la importancia de la consideración individual por las personas, lo que implica un trabajo del líder para apoyar, estimular y orientar a los seguidores (Fernet et al., 2015).

El Liderazgo Transaccional, se destaca por generar un acuerdo explícito con los seguidores respecto de las metas y las expectativas de recompensas, a lo que se suma el monitoreo y control de los subordinados por parte del líder a partir de mediciones respecto de las metas convenidas (por ejemplo, Masa'deh et al., 2016). Las características que distinguen al liderazgo transaccional son las siguientes: recompensa contingente, que implica que el líder define qué hacer, cómo hacerlo y las recompensas asociadas a cada nivel de logro (Gilbert et al., 2016); y gestión por excepción, que se refiere al proceso mediante el cual los líderes monitorean el progreso de los seguidores y los redireccionan hacia el camino correcto cuando existen desviaciones significativas (Birasnav, 2014). Finalmente, el estilo "Laissez faire" se refiere a que el conductor trata de evitar las decisiones y las acciones que le hagan responsable por los resultados (Furtner et al., 2013) Dicho de modo claro, en este caso el liderazgo no se ejerce por parte de la autoridad, y se produce la ausencia física o psicológica de la dirección en momentos críticos, evitando la conducción y el control de los seguidores (Skogstad et al., 2014).

La literatura que sirve de base para relacionar los estilos de liderazgo con la calidad de las instituciones de educación deja de manifiesto que los líderes deberían crear un ambiente de aprendizaje social y emocional para generar los resultados académicos esperados (Jyoti y Bhau, 2016); impactando además sobre el desempeño institucional (Wahab et al., 2016). Más aún, el estilo de liderazgo transformacional parece ser funcional a un ambiente de trabajo orientado al aseguramiento de la calidad académica (Chaudhuri et al. 2016). Además, el liderazgo puede impactar la calidad de la docencia mediante la generación de un ambiente de trabajo académico en el cual los profesores se sienten comprometidos y partícipes de los resultados logrados (Scott y Scott, 2016). En cambio, el estilo laissez faire genera efectos negativos en la actuación (Skogstad et al., 2007) y finalmente en el desempeño del equipo (Spinelli, 2006). Ciertamente, es posible indicar que los estilos de liderazgo pueden impactar en la calidad académica mediante múltiples dimensiones, entre las cuales resulta posible considerar el clima laboral. 


\section{Clima de las unidades académicas}

El clima organizacional en las unidades académicas puede ser conceptualizado como un conjunto integrado de percepciones individuales y colectivas que tiene el cuerpo académico sobre su unidad de trabajo referido a variables psicosociales, laborales y organizacionales, que influyen sobre el comportamiento de los miembros de las respectivas unidades y sobre sus resultados (Schulz, 2013). Un clima organizacional positivo es aquel en el que la integración de los académicos es sólida, donde además el grado de satisfacción y reconocimiento de los académicos es alto (Rabossi, 2017). Por cierto, el clima organizativo y la cultura laboral pueden tener efectos profundos y positivos en el aprendizaje de los estudiantes, impactando sobre los resultados académicos (Ponnuswamy y Manohar, 2016), incluso en la integridad del trabajo académico (Fanelli, 2015), y generando efectos sobre toda la comunidad educativa (Rice y Alexakis, 2015). Al respecto, Stetzer et al. (1997) señalan que el clima organizacional se configura a partir de 4 dimensiones: clima orientado a la excelencia; clima orientado al servicio en términos de proporcionar la mejor calidad posible a un determinado nivel de precio; clima orientado a la cooperación en el cual la colaboración entre los miembros del equipo; clima orientado a la confianza.

El clima orientado a la excelencia se caracteriza por un compartimiento colectivo tendiente al logro de estándares excepcionales, sólo alcanzables por una elite de grupos u organizaciones. Por su cuenta, el clima orientado al servicio se distingue porque el equipo se focaliza en proporcionar el mayor beneficio posible a los usuarios de acuerdo con el precio asociado a la prestación respectiva. De la misma manera, el clima orientado a la colaboración muestra una conducta colectiva de cooperación mutua entre los miembros del equipo. Finalmente, el clima orientado a la confianza se sustenta en un esfuerzo colectivo por generar un ambiente de franqueza y sinceridad en las relaciones de trabajo.

Una revisión del acervo de conocimientos permite visualizar que en el campo de la educación superior, se ha descubierto que un clima orientado a la excelencia puede impactar en los resultados institucionales (Eryılmaz et al., 2016); así como en la sustentabilidad de los programas y en la calidad de los titulados (Dillon y Smith, 2017). A su turno, un clima orientado al servicio puede generar ventaja competitiva en las instituciones de educación superior (Yeo, 2014), focalizando los esfuerzos en el aprendizaje de los estudiantes (Barth et al., 2014), y generando incluso una extensión de su accionar hacia la comunidad (Holland, 2016). Por su parte, un clima orientado a la cooperación produce integración y un ambiente proclive al diálogo (Hernández et al., 2015), estimulando impactos en los procesos de enseñanza (Colvin, 2015), en los resultados (Miller, 2016) y en la sustentabilidad del quehacer de la unidad académica (Wooltorton et al., 2015). Finalmente, el clima orientado a generar confianza entre los miembros de un equipo académico tiene efectos en los niveles de eficacia logrados por la unidad académica (Daly et al., 2015), favoreciendo el alto rendimiento y la alta productividad académica (Tschannen-Moran y Gareis, 2015)

\section{Calidad de las carreras universitarias}

En Chile la calidad de las titulaciones universitarias se rige por un sistema de evaluación de la calidad asociada a procesos de acreditación, a través, de los cuales una agencia externa determina si la respectiva titulación acredita o no, y de hacerlo se define un número de años que puede llegar a un máximo de 7 años. De esta manera, los años de acreditación se constituyen en la principal "proxy" de la calidad del sistema de educación terciaria en Chile (por ejemplo, Rodríguez Ponce et al., 2012). Los procesos de evaluación de la calidad de las titulaciones universitarias en Chile han focalizado su medición en los propósitos e institucionalidad, las condiciones de operación, los procesos formativos, los resultados y la capacidad de autorregulación (Comisión Nacional de Acreditación, 2016).

En el caso de Chile, los propósitos y la institucionalidad proporcionan el marco en el cual la titulación universitaria se implementa y por lo tanto constituye un criterio de evaluación que pretende dar cuenta si el programa académico dispone o no de una clara definición de sus objetivos y metas, junto a políticas y mecanismos que velen y garanticen el cumplimiento de tales propósitos. De tal forma, esta evaluación considera la integridad, es decir, la corrección, ética y robustez académica en que se sustenta la titulación. Complementariamente la consistencia entre el perfil de egreso y el plan de estudios, y su alineamiento con los requerimientos del mercado, son otro elemento a considerar por este criterio de evaluación. La importancia y pertinencia de estas variables ha sido reconocida en múltiples estudios de acuerdo con la evidencia internacional (por ejemplo, Yin et al., 2016).

Otro criterio clave de evaluación de la calidad de las carreras universitarias son las condiciones de operación referidas la administración y organización, el personal docente y su capacidad de creación e investigación, la infraestructura y recursos para el aprendizaje. La evidencia internacional es plenamente consistente con este criterio (por ejemplo, Clark et al., 2016;). La calidad de los procesos formativos es otro criterio de evaluación en el sistema chileno, y su importancia ha sido relevada internacionalmente. En efecto, Dike y Umegboro 
(2015) descubren que los procesos de enseñanza generan diferencias en el grado de aprendizaje de los estudiantes, por cuanto de acuerdo con los objetivos educativos y los perfiles de los alumnos existen procesos que son más apropiados que otros para lograr el aprendizaje. Equivalentemente, Wang et al. (2016) revelan que los métodos de enseñanza tienen impactos significativos en la percepción de autonomía, en la autoeficacia, y en la autorregulación de los educandos, todo lo cual tiene efectos posteriores en el nivel de logro académico. Los procesos formativos trascienden la sola interacción en clases entre profesores y estudiantes e incorporan el contexto social e institucional, descubriéndose que un resultado de los procesos de formación es el compromiso y la motivación para seguir aprendiendo (Boekarts, 2016).

Finalmente, los resultados y la capacidad de autorregulación evalúan las tasas de titulación, el período efectivo de duración de los programas académicos en comparación con su tiempo ideal, así como la inserción de los egresados en el mercado laboral en términos de la tasa de empleo y remuneraciones percibidas. Además, se considera la capacidad del programa para tomar las medidas correctivas pertinentes durante las diferentes fases del ciclo de calidad. La evidencia internacional es consistente con este criterio de evaluación, al reconocer que la formación universitaria no sólo implica aprehender y empoderarse de contenidos, sino que también de actitudes, habilidades y competencias para resolver los desafíos de una disciplina en el ámbito laboral (Biesta, 2015), la tasa de titulación es una medida objetiva de la eficacia de una titulación (Graham et al., 2013), así como su duración efectiva.

\section{ESTUDIO EMPÍRICO}

En esta sección se presenta la naturaleza de la investigación (exploratoria) con la metodología de los participantes, el procedimiento de recolección, las dimensiones y medidas con el respectivo análisis de los datos.

\section{Una aproximación de tipo exploratoria}

Este estudio empírico se aborda como una investigación de naturaleza exploratoria, por lo que se trabaja en una pequeña escala para identificar mediante datos empíricos aspectos fundamentales que proporcionen una visión general, de tipo aproximativo, de las percepciones de los líderes de las unidades académicas que prestan servicios esenciales a un conjunto de carreras universitarias, en lo referente a las relaciones entre: estilos de liderazgo, clima organizacional y calidad de las carreras.

Dicho análisis de tipo exploratorio es pertinente y necesario toda vez que la temática no ha sido suficientemente abordada para el caso de países emergentes como Chile y, por lo tanto, las variables exploradas no han sido indagadas en profundidad en un marco de sus interrelaciones, e incluso las medidas pueden requerir una validación empírica para demostrar su fiabilidad para estas relaciones específicas.

Por consiguiente, en esta investigación puntual se procura validar las variables y sus medidas, analizar su fiabilidad, y establecer una primera mirada acerca de la pertinencia de las relaciones entre: los estilos de liderazgo (transformacional, transaccional, laissez faire); el clima organizativo (orientado a la excelencia; orientado al servicio; orientado a la cooperación; orientado a la confianza); y la calidad de las titulaciones (años de acreditación).

\section{Participantes y procedimiento de recolección}

Se aplicó un cuestionario a 56 líderes formales de unidades académicas elegidos de manera no probabilística, cuyas unidades prestan servicios esenciales a determinadas titulaciones universitarias de 4 instituciones chilenas. En efecto, el requisito que debían cumplir quienes respondieron el cuestionario fue ser director o jefe de una unidad académica que prestase servicios centrales a una carrera universitaria (por ejemplo, el departamento de ingeniería mecánica que presta servicios a la carrera de Ingeniería Civil Mecánica; o el departamento de Psicología que presta servicios a la carrera de Psicología), con una experiencia mínima de 2 años en tales funciones y con una modalidad contractual de jornada completa. Finalmente se obtuvieron 40 respuestas correspondientes a carreras en el ámbito de las ciencias, ingeniería, negocios, ciencias de la salud y humanidades. La tasa de respuesta es del $71,4 \%$, equivalente al de otras investigaciones exploratorias similares (por ejemplo, Araneda-Guirriman et al., 2016).

\section{Dimensiones y medidas}

La dimensión liderazgo considera las variables: (1) liderazgo transformacional, (2) liderazgo transaccional y (3) estilo laissez faire. Los estilos de liderazgo emplean una adaptación del Multiple Leadership Questionnarie (MLQ 5X corto) de Bass y Avolio (1995), y se utiliza a su vez una escala Likert de 1 a 7. La dimensión clima organizativo de las unidades académicas considera las variables: (1) orientación a la excelencia, (2) 
orientación al servicio, (3) orientación a la cooperación, (4) orientación a la confianza adaptados de Stetzer et al. (1997), y se emplea una escala de Likert de 1 a 7 . Finalmente, se utiliza como proxy de calidad de las titulaciones el número de años de acreditación reconocidos oficialmente por la Comisión Nacional de Acreditación de Chile al 31 de diciembre del año 2016, que varía desde 0 (no acreditación) hasta 7 (máxima acreditación). En el Anexo $\mathrm{N}^{\circ} 1$ se adjunta el cuestionario empleado. La Tabla 1 resume los índices de fiabilidad de cada una de las variables de la investigación.

Tabla 1: Índices de fiabilidad de las variables

\begin{tabular}{lc}
\hline \multicolumn{1}{c}{ Variables } & Fiabilidad \\
\hline Liderazgo Transformacional & 0,651 \\
Liderazgo Transaccional & 0,917 \\
Estilo Laissez Faire & 0,967 \\
Cultura orientada a la excelencia & 0,727 \\
Cultura orientada al servicio & 0,666 \\
Cultura orientada a la cooperación & 0,692 \\
Cultura orientada a la confianza & 0,691 \\
\hline
\end{tabular}

\section{Análisis de datos}

Los datos recogidos mediante el cuestionario fueron ingresados en una base de datos empleando el sistema IBM SPSS 23.0 para la realización del análisis estadístico. Se analizaron las medias y desviaciones estándar de cada dimensión junto a las correlaciones de Pearson, y finalmente se analiza un modelo de regresión múltiple, mediante pasos sucesivos, considerando los efectos directos e indirectos de los estilos de liderazgo sobre la calidad de las titulaciones, así como la influencia del clima organizativo sobre la calidad de las instituciones. La idea del análisis de datos más que generar valores específicos consiste en descubrir relaciones entre las variables sustentadas en datos empíricos para cumplir con la lógica de un estudio de naturaleza exploratoria.

\section{RESULTADOS}

Los resultados se generan a partir del análisis econométrico de los datos, pero en ningún caso debe perderse de vista que esta es una investigación exploratoria que trabaja con una muestra no probabilística y en pequeña escala. Por lo tanto, estos resultados no son extrapolables y tienen el propósito de proporcionar una visión general de las relaciones analizadas, las que son válidas para la muestra específica empleada, considerando en todo caso las restricciones propias del estudio asociadas a: un número de datos reducido y la selección no aleatoria de los participantes.

Los estadísticos descriptivos revelan que los años promedio de acreditación de las titulaciones analizadas es de 4,52 años equivalente al promedio nacional de años de acreditación para carreras profesionales en Chile (promedio nacional $=4,50$ ). A su vez, cada una de las variables independientes tiene valores promedios que distan del ideal teórico (7,00 ó 1,00 según corresponda) y tienen una desviación típica mayor que cero. Los resultados se presentan en la Tabla 2. Asimismo, se descubre que la calidad de las titulaciones universitarias se correlaciona de manera positiva y significativa con el estilo de liderazgo transformacional $($ Corr. 0,$778 ; p<$ $0,01$ ), el liderazgo transaccional (Corr. 0,596; $p<0,01)$, con el clima orientado a la excelencia (Corr. 0,$80 ; p$ $<0,01$ ), y con el clima orientado al servicio (Corr. 0,709; $p<0,01$ ). De igual modo, la calidad de las titulaciones universitarias muestra una correlación negativa y significativa con el estilo de liderazgo laissez faire $(p<0,01)$. El clima orientado a la cooperación y el clima orientado a la confianza no tiene un nivel de correlación significativo con la calidad de las titulaciones universitarias. Naturalmente, en un estudio de naturaleza exploratoria lo relevante es la aproximación general, más que las significancia estadística específica, por lo que estos resultados sugieren que: existe evidencia empírica para relacionar los estilos de liderazgo con el clima en las unidades académicas. La Tabla 3 exhibe las correlaciones de Pearson a las que se hizo alusión previamente:

Tabla 2: Estadísticos descriptivos

\begin{tabular}{|l|c|c|c|}
\hline & Media & Desviación típica & N \\
\hline Añosacreditacion & 4,5250 &, 71567 & 40 \\
\hline Culturaexcelencia & 4,6813 &, 70253 & 40 \\
\hline Culturaservicio & 4,5938 &, 70866 & 40 \\
\hline
\end{tabular}


Tabla 2 (continuación)

\begin{tabular}{|l|l|l|l|}
\hline Culturacooperacion & 3,2063 &, 77602 & 40 \\
\hline Culturaconfianza & 3,8000 &, 87560 & 40 \\
\hline Transformacional & 4,8008 &, 62314 & 40 \\
\hline Transaccional & 5,1550 &, 95245 & 40 \\
\hline Laissezfaire & 2,6425 &, 97241 & 40 \\
\hline
\end{tabular}

Tabla 3: Correlaciones de Pearson **. La correlación es significativa al nivel 0,01 (bilateral); *. La correlación es significante al nivel 0,05 (bilateral).

\begin{tabular}{|c|c|c|c|c|c|c|c|c|}
\hline & $\begin{array}{l}\text { Lid. } \\
\text { Transf. }\end{array}$ & $\begin{array}{l}\text { Lid. } \\
\text { Trans. }\end{array}$ & $\begin{array}{l}\text { Laissez } \\
\text { faire }\end{array}$ & $\begin{array}{l}\text { Clima } \\
\text { excelencia }\end{array}$ & $\begin{array}{l}\text { Clima } \\
\text { servicio }\end{array}$ & $\begin{array}{l}\text { Clima } \\
\text { cooperación }\end{array}$ & $\begin{array}{l}\text { Clima } \\
\text { confianza }\end{array}$ & $\begin{array}{l}\text { Calidad } \\
\text { titulación }\end{array}$ \\
\hline Lid. Transf. & 1 &, $481^{* *}$ &,$- 500^{\star *}$ &, $867^{\star *}$ &, $792^{\star \star}$ &,- 100 &, 178 &, $778^{\star *}$ \\
\hline Lid. Trans. &, $481^{* *}$ & 1 &,$- 709^{* *}$ &, $543^{* *}$ & $349^{*}$ &, 112 &, 068 &, $596^{\star \star}$ \\
\hline Laissez faire &,$- 500^{\star \star}$ &,$- 709^{* *}$ & 1 &,$- 474^{\star *}$ &,- 311 &,- 150 &, 127 &,$- 575^{\star *}$ \\
\hline $\begin{array}{l}\text { Clima } \\
\text { excelencia }\end{array}$ &, $867^{\star \star}$ &, $543^{\star *}$ &,$- 474^{\star \star}$ & 1 &, $882^{* \star}$ &, 006 & , 110 &, $800^{* \star}$ \\
\hline $\begin{array}{l}\text { Clima } \\
\text { servicio }\end{array}$ &, $792^{* *}$ & $349^{*}$ &,- 311 & ,882** & 1 & , 104 & ,201 & ,709** \\
\hline $\begin{array}{l}\text { Clima } \\
\text { coopera. }\end{array}$ &,- 100 & 112, &,- 150 & ,006 & , 104 & 1 &,- 008 & ,019 \\
\hline $\begin{array}{l}\text { Clima } \\
\text { confianza }\end{array}$ & , 178 & ,068 & , 127 & , 110 & ,201 &,- 008 & 1 & , 131 \\
\hline $\begin{array}{l}\text { Calidad } \\
\text { titulación }\end{array}$ &, $778^{* *}$ &, $596^{\star *}$ &,$- 575^{\star *}$ &, $800^{* *}$ &, $709^{* *}$ & ,019 & ,131 & 1 \\
\hline
\end{tabular}

Ahora bien, al definir como variable independiente la calidad de las carreras (años de acreditación) y como variables independientes los estilos de liderazgo (transformacional, transaccional, laissez faire) junto con la orientación del clima laboral (excelencia, servicio, cooperación, confianza), empleando el método de pasos sucesivos, se encuentra que el estilo de liderazgo laissez faire y el clima orientado a la excelencia son las variables que, por sí solas, explican la proporción más significativa de la varianza de los años de acreditación. En efecto, ambas variables en conjunto explican el $67,3 \%$ de la varianza en la calidad de las titulaciones, lo que es estadísticamente significativo (Test $F=41,106 ; p<0,01$ ). Las Tablas 4 y 5 muestran estos resultados.

Tabla 4: Capacidad explicativa del modelo Laissez Faire, Clima excelencia

\begin{tabular}{|c|c|c|c|c|c|c|c|c|}
\hline \multirow[t]{2}{*}{$\mathrm{R}$} & \multirow{2}{*}{$\begin{array}{c}\mathrm{R} \\
\text { cuadrado }\end{array}$} & \multirow{2}{*}{$\begin{array}{l}\mathrm{R} \text { cuadrado } \\
\text { corregida }\end{array}$} & \multirow{2}{*}{$\begin{array}{l}\text { Error típ. de } \\
\text { la estimación }\end{array}$} & \multicolumn{5}{|c|}{ Estadísticos de cambio } \\
\hline & & & & Cambio en $\mathrm{R}^{2}$ & Cambio en $\mathrm{F}$ & gl1 & gl2 & Sig. Cambio en F \\
\hline 830 & ,690 & ,673 & ,40934 & ,049 & 5,848 & 1 & 37 & ,021 \\
\hline
\end{tabular}

Tabla 5: Análisis de varianza ANOVA. Variables predictoras: (Constante), CULTURACALIDAD, LAISSEZFAIRE

\begin{tabular}{|l|l|l|c|l|l|}
\hline Modelo & Suma de cuadrados & gl & Media cuadrática & F & Sig. \\
\hline Regresión & 13,775 & 2 & 6,888 & 41,106 &, $000^{c}$ \\
\hline Residual & 6,200 & 37 &, 168 & & \\
\hline Total & 19,975 & 39 & & & \\
\hline
\end{tabular}

Por tanto, las variables explicativas directas de la calidad de las carreras universitarias en esta investigación exploratoria serían: el estilo laissez faire que tiene un impacto negativo y significativo sobre los años de acreditación (Test $\mathrm{t}=-2,418 ; \mathrm{p}<0,021$ ); y el clima orientado a la calidad que tiene un efecto positivo $\mathrm{y}$ significativo (Test $t=6,547 ; p<0,01$ ). La siguiente Tabla 6 muestra estos resultados:

Tabla 6: Significancia de las variables independientes

\begin{tabular}{lcrrrr}
\hline \multirow{2}{*}{ Modelo } & \multicolumn{2}{c}{ Coeficientes no estandarizados } & $\begin{array}{c}\text { Coeficientes } \\
\text { tipificados }\end{array}$ & $\mathrm{t}$ & Sig. \\
\cline { 2 - 4 } & \multicolumn{1}{c}{$\mathrm{B}$} & Error típ. & Beta & & \\
\hline (Constante) & 1,766 &, 622 &, 007 &, 842 \\
Clima Excelencia &, 694 &, 106 &, 681 & 6,547 &, 000 \\
Laissez Aire &,- 185 &, 077 &,- 252 & $-2,418$ &, 021 \\
\hline
\end{tabular}


Los resultados encontrados sugieren la existencia de una evidencia empírica que muestra que el clima orientado a la excelencia tiene importancia para favorecer la calidad de las carreras; en tanto que el estilo de liderazgo laissez faire o evitador actúa en detrimento de la calidad de las titulaciones universitarias. Luego, al relacionar el clima orientado a la excelencia con los estilos de liderazgo (transformacional, transaccional, laissez faire), se halla en la muestra que el estilo de liderazgo transformacional explica el $74,5 \%$ de la varianza del clima orientado a la excelencia (Test $F=115,108 ; p<0,01$ ), según Tablas 7,8 y 9 :

Tabla 7: Capacidad explicativa del modelo

\begin{tabular}{|c|c|c|c|c|c|c|c|c|c|}
\hline \multirow[t]{2}{*}{ Modelo } & \multirow[t]{2}{*}{$\mathrm{R}$} & \multirow[t]{2}{*}{ R cuadrado } & \multirow{2}{*}{$\begin{array}{l}\text { R cuadrado } \\
\text { corregida }\end{array}$} & \multirow{2}{*}{$\begin{array}{l}\text { Error típ. de la } \\
\text { estimación }\end{array}$} & \multicolumn{5}{|c|}{ Estadísticos de cambio } \\
\hline & & & & & $\begin{array}{l}\text { Cambio en } \\
\mathrm{R} \text { cuadrado }\end{array}$ & $\begin{array}{c}\text { Cambio en } \\
F\end{array}$ & gl1 & gl2 & $\begin{array}{c}\text { Sig. } \\
\text { Cambio en } \\
\text { F }\end{array}$ \\
\hline 1 & $867^{a}$ & ,752 & 745 & ,35457 & 752 & 115,108 & 1 & 38 &, 000 \\
\hline
\end{tabular}

Tabla 8: Análisis de varianza ANOVA

\begin{tabular}{lccccc}
\hline Modelo & Suma de cuadrados & Gl & Media cuadrática & F & Sig. \\
\hline Regresión & 14,471 & 1 & 14,471 & 115,108 &, $000^{\mathrm{b}}$ \\
1 Residual & 4,777 & 38 &, 126 & & \\
Total & 19,248 & 39 & & & \\
\hline a. Variable dependiente: CLIMAEXCELENCIA & & & \\
b. Variables predictoras: (Constante), TRANSFORMACIONAL & &
\end{tabular}

Tabla 9: Significancia de las variables independientes

\begin{tabular}{|c|c|c|c|c|c|c|}
\hline \multicolumn{2}{|c|}{ Modelo } & \multicolumn{2}{|c|}{$\begin{array}{l}\text { Coeficientes no } \\
\text { estandarizados }\end{array}$} & \multirow{2}{*}{$\begin{array}{c}\text { Coeficientes } \\
\text { tipificados } \\
\text { Beta }\end{array}$} & \multirow[t]{2}{*}{$\mathrm{t}$} & \multirow[t]{2}{*}{ Sig. } \\
\hline & & $\mathrm{B}$ & Error típ. & & & \\
\hline 1 & $\begin{array}{l}\text { (Constante) } \\
\text { Transformacional }\end{array}$ & $\begin{array}{r}-, 012 \\
, 978 \\
\end{array}$ & $\begin{array}{l}, 441 \\
, 091 \\
\end{array}$ & ,867 & $\begin{array}{r}-, 026 \\
10,729 \\
\end{array}$ & $\begin{array}{l}, 979 \\
, 000\end{array}$ \\
\hline
\end{tabular}

En consecuencia, en esta muestra analizada el clima orientado a la excelencia está incidido de manera positiva y significativa por el estilo de liderazgo transformacional (Test $t=10,729 ; p<0,01$ ), lo que implica que el estilo de liderazgo transformacional tendría un efecto indirecto sobre la calidad de las carreras universitarias. La importancia desde la perspectiva de una investigación de naturaleza exploratoria es que se genera evidencia empírica que respalda la existencia de una relación específica entre estilos de liderazgo y clima académico y sus posteriores efectos sobre la calidad de las carreras. La figura 1 muestra los resultados exploratorios, empleado una muestra elegida por conveniencia (facilidad de acceso) y en pequeña escala, obtenidos en esta investigación.

Nótese que más importante que los valores específicos obtenidos mediante un análisis de regresión, por pasos sucesivos, lo fundamental es que trabajando en pequeña escala se ha encontrado evidencia empírica que respalda relaciones entre: estilos de liderazgos/ clima académico/ calidad de las carreras. De este modo, los resultados sugieren que no se puede descuidar las actuaciones que configuran un liderazgo transformacional para lograr un clima de excelencia, el cual a su vez parece tener relación significativa con la calidad de las carreras universitarias. En tanto el estilo laissez faire exhibe efectos negativos sobre la calidad de las titulaciones.

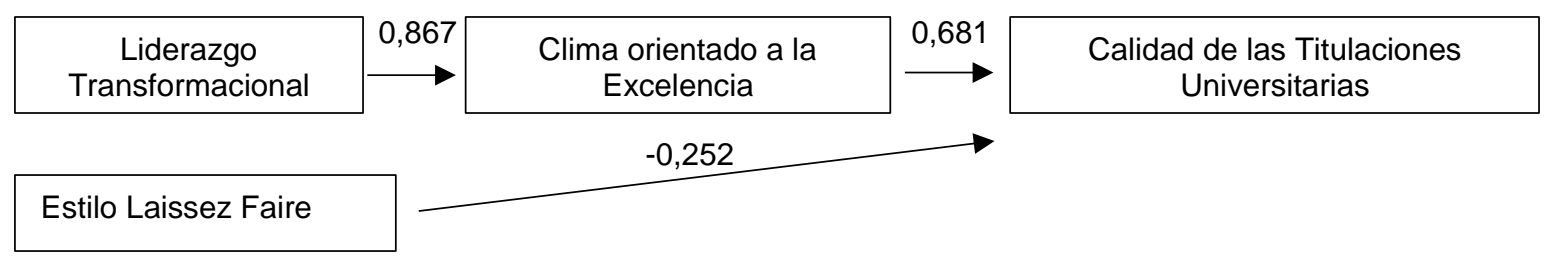

Fig. 1. Resultados exploratorios

\section{DISCUSIÓN}

Los resultados revelan evidencia para sustentar empíricamente la existencia de una relación entre: estilos de liderazgo/ clima organizacional/ calidad de las carreras universitarias. La forma específica en la cual esta relación se configura, en la muestra analizada, es a través del impacto directo y negativo del estilo de liderazgo laissez faire sobre los años de acreditación de las titulaciones o carreras universitarias y a partir del efecto altamente significativo y positivo del clima orientado a la excelencia sobre los años de acreditación de las carreras o titulaciones universitarias. 
En efecto, en la muestra analizada, por cada punto porcentual que aumentase el estilo laissez faire de los directivos en las unidades académicas, la calidad de las titulaciones universitarias disminuye 0,252 puntos porcentuales. Más allá de los valores específicos encontrados lo relevante es la evidencia de una relación negativa entre el estilo laissez y los años de acreditación de las carreras universitarias. Este hallazgo cuenta con soporte en el estado del arte ya que los líderes laissez faire evitan tomar decisiones y omiten ejercer acciones que les hagan responsables por los resultados (por ejemplo, Furtner et al., 2013), lo que conlleva a una ausencia de dirección y control en momentos críticos (Skogstad et al., 2014). Así las cosas, no es de extrañar que un estilo laissez faire se asocie con conductas menos proclives a lograr resultados deseables (Skogstad et al., 2007) y con un menor desempeño del equipo de trabajo en el cumplimiento de sus metas (Spinelli, 2006).

Por su parte, el estilo de liderazgo transformacional tiene un impacto directo sobre el clima orientado a la excelencia. Ciertamente, en la muestra analizada, un aumento de un punto porcentual en el liderazgo transformacional implicaría un aumento de 0,867 puntos porcentuales en el clima orientado a la calidad. Pero, por sobre valores específicos, la relevancia de este estudio exploratorio es que se encuentra evidencia de una relación positiva entre liderazgo transformacional y el clima orientado a la excelencia. En todo caso, este hallazgo tiene evidencia en el acervo de conocimientos por cuanto el líder transformacional genera metas desafiantes y logra que sus seguidores se sumen al logro de tales metas, construyendo un clima basado en los valores institucionales, en el que se releva la contribución de cada uno en los logros del equipo (por ejemplo, Cheng et al., 2016). Dicho estilo impacta en la construcción de las percepciones individuales y colectivas que tiene el cuerpo académico sobre su unidad de trabajo y, en definitiva, en el clima y el comportamiento de los miembros de las unidades y sus resultados (por ejemplo, Hussein et al., 2014).

Complementariamente, se encuentra que un aumento de un punto porcentual en el clima orientado a la excelencia tendría un efecto de 0,681 puntos porcentuales en la calidad de las carreras universitarias. Nuevamente, debe señalarse que más allá de los valores encontrados la relevancia del hallazgo estriba en que existe evidencia para relacionar la orientación hacia la excelencia en el clima laboral académico con la calidad de las carreras. Este es un resultado plenamente consistente con la evidencia internacional, ya que múltiples investigaciones han probado que un clima orientado a la calidad incide en los resultados institucionales (Eryılmaz et al., 2016); y de los propios programas académicos impactando sobre la calidad de los titulados (Dillon y Smith, 2017).

Además, dado que en la muestra analizada, el estilo transformacional tiene efectos sobre el clima orientado a la calidad y esta última variable se relaciona con la calidad de las titulaciones universitarias existiría un efecto indirecto, pero positivo del liderazgo transformacional sobre los años de acreditación de las carreras. Efectivamente, una variación en un punto porcentual en el estilo de liderazgo transformacional impactaría en 0,590 puntos porcentuales en la calidad de las titulaciones. Los hallazgos de la investigación configuran una primera aproximación al análisis de las relaciones entre estilos de liderazgo/clima organizativo/ calidad de las titulaciones, en el campo de la educación superior en países emergentes como Chile. Se cumple, por tanto, con el objetivo de la investigación que consiste precisamente en explorar las relaciones antes descritas, proporcionándose a la vez valiosa evidencia empírica y elementos teóricos para una construcción de teoría, cuya verificación sería materia de otra investigación cuantitativa empleando una muestra representativa tal que permita realizar inferencia estadística.

Además, es muy importante indicar que la eventual relación de causalidad de las variables es una tarea adicional a demostrar dado que los cálculos realizados parten de un sustento teórico y tienen la finalidad de descubrir relaciones potenciales; sin embargo, una validación empírica requeriría de un estudio en profundidad que permitiría identificar el sentido de dependencia e independencia de las relaciones exploradas.

\section{CONCLUSIONES}

De acuerdo a la investigación realizada, al análisis y discusión, es posible derivar un conjunto de conclusiones principales: 1) Las variables y medidas empleadas en esta investigación exploratoria son suficientemente válidas (teóricamente) y fiables (empíricamente), aunque desde luego admiten mejoras particularmente aquellas cuyo Alpha de Cronbach está en el rango entre 0,651 y 0,692;2) Existe evidencia relevante de la pertinencia de plantear una relación entre: estilos de liderazgo/ clima organizacional/ calidad de las carreras universitarias, lo cual constituye un hallazgo novedoso en el campo de la educación superior en países emergentes; 3) El estilo de liderazgo laissez faire no es inocuo, sino que es potencialmente negativo para lograr mayores niveles de calidad en las titulaciones universitarias. Esto es el dejar hace y dejar pasar no tiene impacto nulo sino que daña la calidad del quehacer académico; 4) El liderazgo transformacional, en la muestra analizada, no tiene un impacto directo sobre la calidad de las titulaciones, pero su impacto es indirecto y muy significativo incluso a nivel de cuantía (0,59 puntos porcentuales). A su turno, el liderazgo transformacional es el principal determinante de la generación de un clima orientado a la excelencia; 5) Un 
clima orientado a la excelencia tiene una incidencia positiva y significativa sobre la calidad de las carreras o titulaciones universitarias, constituyéndose en la variable más relevante en significación y en la magnitud del impacto que puede generar en la muestra analizada; 6) Una investigación de naturaleza exploratoria tiene la limitación que sus resultados no son extrapolables bajo la lógica de inferencia estadística, aunque los mismos pueden ser valiosos en una etapa preliminar de construcción de teoría porque de todas formas es posible mediante inducción analítica desprender los elementos críticos que pueden tener mayor relevancia sobre la calidad de las titulaciones universitarias; 7) A partir de este estudio emerge la necesidad de realizar futuras investigaciones en las que mediante un estudio de casos en profundidad se validen empíricamente las relaciones causales de estas variables y seguidamente mediante un estudio de corte transversal se estudie toda la población de carreras universitarias, seleccionando aleatoriamente una muestra de las mismas, analizando los resultados mediante ecuaciones estructurales y técnicas de inferencia estadística.

\section{AGRADECIMIENTOS}

Esta investigación ha sido posible gracias al financiamiento de la Comisión Nacional del Investigación Científica y Tecnológica de Chile mediante los proyectos FONDECYT Regular 1140026 y 1140027. Los autores agradecen los comentarios y sugerencias de la Mag. Carmen Araneda-Guirriman y la Dra. Liliana Pedraja Rejas por su revisión y valiosas observaciones.

\section{REFERENCIAS}

Alonderiene, R. y M. Majauskaite: Leadership style and job satisfaction in higher education institutions. International Journal of Educational Management, 30(1), 140-164 (2016)

Araneda-Guirriman, C.A., N.A., Neumann-González, L.M. Pedraja-Rejas y E.R. Rodríguez-Ponce: Análisis Exploratorio de las Percepciones sobre los Estilos de Liderazgo de los Directivos Universitarios en el Norte de Chile. Formación Universitaria, 9(6), 139-152 (2016)

Barro, R. J., y J. W. Lee: A new data set of educational attainment in the world, 1950-2010. Journal of Development Economics, 104, 184-198 (2013)

Barth, M., M. Adomßent, D. Fischer, S. Richter, y M. Rieckmann: Learning to change universities from within: a service-learning perspective on promoting sustainable consumption in higher education. Journal of Cleaner Production, 62, 72-81 (2014)

Belias, D., E. Velissariou y otros cuatro autores: The Role of Organizational Culture in the Greek Higher Tourism Quality. Strategic Innovative Marketing. Springer, Cham, 65-70 (2017)

Biesta, G.: What is education for? On good education, teacher judgement, and educational professionalism. European Journal of Education, 50(1), 75-87 (2015)

Birasnav, M.: Knowledge management and organizational performance in the service industry: The role of transformational leadership beyond the effects of transactional leadership. Journal of Business Research, 67(8), 1622-1629 (2014)

Boekaerts, M.: Engagement as an inherent aspect of the learning process. Learning and Instruction, 43, 76-83 (2016)

Boni, A. y M. Walker: Universities and Global Human Development: Theoretical and empirical insights for social change (London, Routledge) (2016)

Castells M.: The rise of the network society: The information age: Economy, society, and culture. John Wiley \& Sons (2011)

Cheng, C., T. Bartram, L. Karimi, y S. Leggat: Transformational leadership and social identity as predictors of team climate, perceived quality of care, burnout and turnover intention among nurses. Personnel Review, 45(6), 1200-1216 (2016)

Colvin, J. W.: Peer Mentoring and Tutoring in Higher Education. Exploring Learning \& Teaching in Higher Education, pp. 207-229. Springer Berlin Heidelberg (2015)

Comisión Nacional de Acreditación, Criterios de evaluación de carreras. 16 pp. (2016)

Daly, A. J., N.M. Moolenaar, Y.H. Liou, M. Tuytens, y M. Del Fresno: Why so difficult? Exploring negative relationships between educational leaders: The role of trust, climate, and efficacy. American Journal of Education, 122(1), 1-38 (2015)

Dickinson, E.R. y J.L. Adelson: Choosing Among Multiple Achievement Measures Applying Multitrait-Multimethod Confirmatory Factor Analysis to State Assessment, ACT, and Student GPA Data. Journal of Advanced Academics, 27(1), 4-22. (2016)

Dike, J. W. y K. Umegboro: Teaching variables and students performance on the concept of saponification. British Journal of Education, 3(8), 40-46 (2015)

Dillon, E.W. y J.A. Smith: Determinants of the match between student ability and college quality. Journal of Labor Economics, 35(1), 45-66 (2017)

Duque, L.C.: A framework for analysing higher education performance: students' satisfaction, perceived learning outcomes, and dropout intentions. Total Quality Management \& Business Excellence, 25(1-2), 1-21 (2014)

Ersozlu, A. y E. Saklan: Instructional leadership in higher education: How does it work? British Journal of Education, 4(5), 1-15 (2016) 
Eryılmaz, M. E., Kara, E., Aydoğan, E., Bektaş, O., \& Erdur, D. A.: Quality Management in the Turkish Higher Education Institutions: Preliminary Findings. Procedia-Social and Behavioral Sciences, 229, 60-69 (2016)

Fanelli, D., R. Costas y V. Larivière: Misconduct policies, academic culture and career stage, not gender or pressures to publish, affect scientific integrity. PloS one, 10(6), e0127556, 18 pp. (2015)

Furtner, M. R.U. Baldegger y J.F. Rauthmann: Leading yourself and leading others: Linking self-leadership to transformational, transactional, and laissez-faire leadership. European Journal of Work and Organizational Psychology, 22(4), 436-449 (2013)

Gilbert, S., P. Horsman y E.K. Kelloway: The Motivation for Transformational Leadership Scale: An examination of the factor structure and initial tests. Leadership \& Organization Development Journal, 37(2), 158-180 (2016)

Graham, M. J., J. Frederick, A. Byars-Winston, A.B. Hunter, y J. Handelsman: Increasing persistence of college students in STEM. Science, 341(6153), 1455-1456 (2013)

Hanushek, E. A.: Economic growth in developing countries: The role of human capital. Economics of Education Review, 37, 204-212 (2013)

Henkel, T. G.: The Relationship Between Transformational Leadership Styles and University Adjunct Faculty Work Engagement. Embry-Riddle Aeronautical University. 22 pp. (2016)

Hernández, K. A. C., F.W. Ngunjiri, y H. Chang: Exploiting the margins in higher education: A collaborative autoethnography of three foreign-born female faculty of color. International Journal of Qualitative Studies in Education, 28(5), 533-551 (2015)

Holland, B. A.: Factors and Strategies That Influence Faculty Involvement in Public Service. Journal of Higher Education Outreach and Engagement, 20(1), 63-71 (2016)

Horn, A. S., y G. Lee: The reliability and validity of using regression residuals to measure institutional effectiveness in promoting degree completion. Research in Higher Education, 57(4), 469-496 (2016)

Hussein, N., A. Mohamad, F. Noordin y N.A, Ishak: Learning organization and its effect on organizational performance and organizational innovativeness: A proposed framework for Malaysian Public Institutions of Higher Education. ProcediaSocial and Behavioral Sciences, 130, 299-304 (2014)

Jyoti, J., y S. Bhau: Empirical investigation of moderating and mediating variables in between transformational leadership and related outcomes: A study of higher education sector in North India. International Journal of Educational Management, 30(6), 1123-1149 (2016)

Long C.S., Thean, L.Y., Ismail W.K. y Jusoh, A: Leadership styles and employees' turnover intention: Exploratory study of academic staff in a Malaysian college. World Applied Science Journal, 19, 575-581 (2012)

Mansour, E. A., Gemeay, E. M., Behilak, S., y Albarrak, M. Factors affecting Attrition Rate among Nursing Students College of Health Sciences, Taibah University, Saudi Arabia. International Journal of Nursing, 3(1), 65-72 (2016)

Masa'deh, R. E., Obeidat, B. Y., y Tarhini, A.: A Jordanian empirical study of the associations among transformational leadership, transactional leadership, knowledge sharing, job performance, and firm performance: A structural equation modelling approach. Journal of Management Development, 35(5), 681-705 (2016)

Miller, B.A.: Assessing organizational performance in higher education. John Wiley \& Sons (2016)

Mukherjee, S.: Leadership network and team performance in interactive contests. Social Networks, 47, 85-92 (2016)

Nussbaum, M. y A. Sen: The Quality of Life. Oxford: Clarendon Press (1993)

Ponnuswamy, I., y H.L. Manohar: Impact of learning organization culture on performance in higher education institutions. Studies in Higher Education, 41(1), 21-36 (2016)

Rabossi, M., Agency Costs in Higher Education: Evaluating an Institution Through a Comprehensive Framework. Higher Education Policy, 30(3), 319-339 (2017)

Rahimi, G. R., S. Khezri, y S. Niknafs: Investigation the relationship of Leadership Styles on managers on productivity Staff Tax Administration of West Azerbaijan province. Human Resource Management, 3(10), 39-43 (2016)

Rice, L., y F. G. Alexakis: Building an Academic Culture of Praxis. Learning in Higher Education, 123 (2015)

Rodríguez-Ponce, E., N. Fleet, L. Pedraja-Rejas y J. Rodriguez-Ponce: Efecto de la calidad de la información sobre la acreditación institucional: un estudio exploratorio en universidades chilenas. Ingeniare. Revista chilena de ingeniería, 20(3), 368-375 (2012)

Skogstad, A., J. Hetland, L. Glasø, S. Einarsen: Is avoidant leadership a root cause of subordinate stress? Longitudinal relationships between laissez-faire leadership and role ambiguity. Work \& Stress, 28(4), 323-341 (2014)

Skogstad, A., S. Einarsen, T. Torsheim, M. S. Aasland, y H. Hetland: The destructiveness of laissez-faire leadership behavior. Journal of occupational health psychology, 12(1), 80-92 (2007)

Solajà, O. M., F.E. Idowu y A.E. James: Exploring the relationship between leadership communication style, personality trait and organizational productivity. Serbian Journal of Management, 11(1), 99-117 (2016) 
Spinelli, R.J.: The applicability of Bass's model of transformational, transactional, and laissez-faire leadership in the hospital administrative environment. Hospital Topics, 84(2), 11-19 (2006)

Stetzer, A., F.P. Morgeson y E.L. Anderson: Organizational climate and ineffectiveness: evidence from 25 outdoor work crew divisions. Journal of Quality Management, 2(2), 251-265 (1997)

Tschannen-Moran, M., y C.R. Gareis: Faculty trust in the principal: An essential ingredient in high-performing schools. Journal of Educational Administration, 53(1), 66-92 (2015)

Vally, S. y E. Motala: Education, Economy \& Society. Pretoria: UNISA Press (2014)

Wahab, S., A. Rahmat, M.S. Yusof, y B. Mohamed: Organization Performance and Leadership Style: Issues in Education Service. Procedia-Social and Behavioral Sciences, 224, 593-598 (2016)

Wang, C.K., Ng, B. L., W.C. Liu y R.M. Ryan: Can being autonomy-supportive in teaching improve students' self-regulation and performance? In Building Autonomous Learners (pp. 227-243). Springer Singapore (2016)

Westrick, P. A., Le, H., Robbins, S. B., Radunzel, J. M., Schmidt, F. L.: College performance and retention: A meta-analysis of the predictive validities of ACT scores, high school grades, and SES. Educational Assessment, 20(1), 23-45 (2015)

Wooltorton, S., A. Wilkinson, P. Horwitz, S. Bahn, J. Redmond y J. Dooley: Sustainability and action research in universities: Towards knowledge for organizational transformation. International Journal of Sustainability in Higher Education, 16(4), 424-439 (2015)

Yeo, R.K., y J. Li: Beyond SERVQUAL: The competitive forces of higher education in Singapore. Total Quality Management \& Business Excellence, 25(1-2), 95-123 (2014)

Yin, H., W. Wang y J. Han: Chinese undergraduates' perceptions of teaching quality and the effects on approaches to studying and course satisfaction. Higher Education, 71(1), 39-57 (2016)

Zeb, A., Saeed, G., Ullah, H., y Rabi, F., Transformational and Transactional Leadership Styles and its Impact on the Performance of the Public Sector Organizations in Pakistan. Abasyn University Journal of Social Sciences, 8(1), 37-46 (2015) 